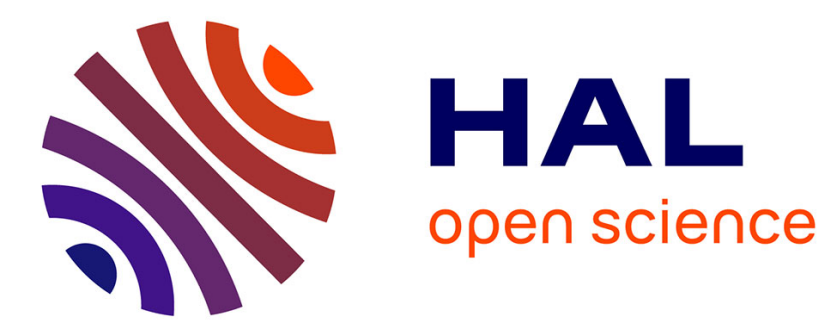

\title{
Nanobubbles and their role in slip and drag
}

Abdelhamid Maali, Bharat Bhushan

\section{To cite this version:}

Abdelhamid Maali, Bharat Bhushan. Nanobubbles and their role in slip and drag. Journal of Physics: Condensed Matter, 2013, 25 (18), pp.184003. 10.1088/0953-8984/25/18/184003 . hal-00825450

\section{HAL Id: hal-00825450 \\ https://hal.science/hal-00825450}

Submitted on 7 Mar 2018

HAL is a multi-disciplinary open access archive for the deposit and dissemination of scientific research documents, whether they are published or not. The documents may come from teaching and research institutions in France or abroad, or from public or private research centers.
L'archive ouverte pluridisciplinaire HAL, est destinée au dépôt et à la diffusion de documents scientifiques de niveau recherche, publiés ou non, émanant des établissements d'enseignement et de recherche français ou étrangers, des laboratoires publics ou privés.

\section{(1)(1) $\$(0)$}

Distributed under a Creative Commons Attribution - NonCommercial - ShareAlikel 4.0 


\title{
Nanobubbles and their role in slip and drag
}

\author{
Abdelhamid Maali ${ }^{1}$ and Bharat Bhushan ${ }^{2,3}$ \\ ${ }^{1}$ Laboratoire Ondes et Matière d'Aquitaine (LOMA), Université Bordeaux I, 351 cours de la Liberation, \\ F-33405 Talence, France \\ ${ }^{2}$ Nanoprobe Laboratory for Bio- and Nanotechnology and Biomimetics $\left(\mathrm{NLB}^{2}\right)$, The Ohio State \\ University, $201 \mathrm{~W}$ 19th Avenue Columbus, OH 43210-1142, USA \\ E-mail: a.maali@1oma.u-bordeaux1.fr and bhushan.2@osu.edu
}

\begin{abstract}
Atomic force microscope images of flat solid surfaces in water reveal that very soft objects can be formed on the surfaces. These objects are nanobubbles of gas with sizes ranging from $10 \mathrm{~nm}$ to several micrometers. The bubbles are stable to dissolution, lasting for several hours. In this paper we review some of the methods that allow their generation and observation using the atomic force microscope. Next, we describe the influence of the bubbles on liquid slip close to a hydrophobic surface. The influence of liquid-gas menisci, formed as a result of nanobubbles being present on the surface, on drag reduction is also discussed. Finally, data of liquid flow probed on bubbles entrapped on microstructured surfaces are presented.
\end{abstract}

\section{Introduction}

Interfaces between surfaces that have a weak intermolecular interaction with liquids (hydrophobic surfaces) are the subject of intensive research. Nanobubbles are found to appear spontaneously at the interface between a liquid and a hydrophobic surface (Wang and Bhushan 2010, Craig 2011, Seddon and Lohse 2011). The bubbles are detected by various techniques, such as tapping mode atomic force microscopy (Maali and Bhushan 2008, Wang and Bhushan 2010), optical microscopy (Karpitschka et al 2012), optical spectroscopy (Zhang et al 2007a, 2008), rapid cryofixation (Switkes and Ruberti 2004), neutron reflectometry (Steitz et al 2003), and $\mathrm{X}$-ray reflectivity measurements (Poynor et al 2006). The bubbles are invoked in some papers as the possible origin of the increase in flow rate of liquids in microchannels (Tretheway and Meinhart 2004). The presence of nanobubbles is used to explain liquid slip at the interface and the long-range attraction between hydrophobic surfaces in water (Tyrrell and Attard 2001, de Gennes 2002, Lauga and Brenner 2004). Some new experimental papers show the opposite (Steinberger et al 2007, 2008, Finger and Johannsmann

${ }^{3}$ Author to whom any correspondence should be addressed.
2011). Actually, there is no precise measurement that probes directly the influence of bubbles on the slip length. To capture correctly the influence of the nanobubbles on the flow close to the wall, a correlation measurement between the density of the bubbles on the surface and the value of slip length is required. Also the relationship between the contact angle between the nanobubbles and the surface and the value of slip length should be studied. To our knowledge the only experiments that measure the influence of interface shape on the slip length and drag force at the nanoscale were performed by Steinberger et al (2007, 2008) and by Maali et al (2012), both of them dealing with bubbles entrapped in water on microstructured surfaces. The surface force apparatus experiments performed by Steinberger et al $(2007,2008)$ clearly showed that the gas bubbles trapped at the solid surface can act as an anti-lubricant and promote high friction. Their measurements show that the bubbles reduced the slip instead of increasing it, and also increased the drag. These measurements can be understood if one takes into account the shape of the nanobubbles, which induces a perturbation of the lines of liquid flow.

In this paper, we first present some of the methods that are used for the generation of bubbles and their observation. In the next section we present a theoretical expression for the hydrodynamic drag force and its relation to the slip 


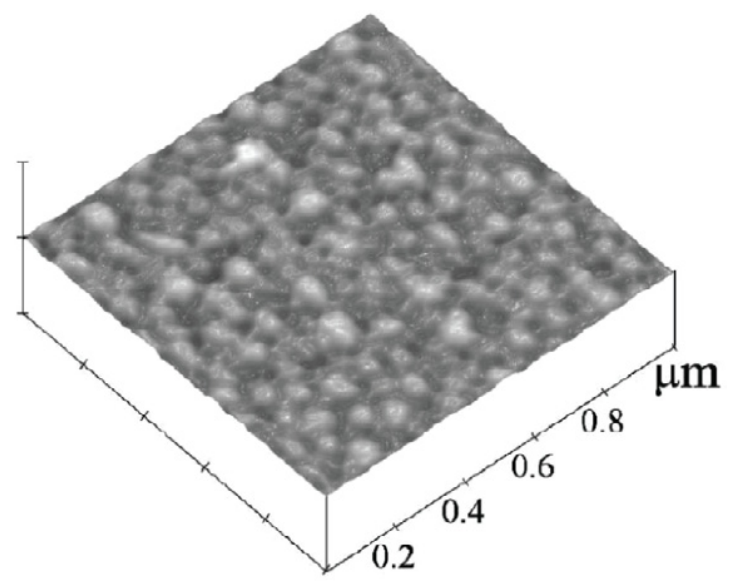

Figure 1. AFM tapping mode images of a $1 \mu \mathrm{m}$ square of a hydrophobic surface in water. The vertical height scale is $30 \mathrm{~nm}$ (Tyrrell and Attard 2001).

length of liquids as well as the effect of nanobubbles on slip length. Next we present the recent results of Steinberger et al (2007, 2008) and Hyväluoma and Harting (2008), which cast some doubt about the role of the bubbles in liquid slip on flat surfaces. In the last section we present an atomic force microscope experiment that confirms the influence of the shape of the meniscus that forms nanobubbles in determining the drag force. This consists of an experiment on the drainage of water between a sphere and a microstructured surface consisting of regularly spaced pillars.

\section{Nanobubbles on flat solid surface}

In this section we present the different methods used to generate nanobubbles on solid surfaces and show representative images. The most popular method for nanobubble studies is tapping mode atomic force microscopy (TMAFM). In this mode the oscillating AFM tip touches the sample intermittently and the force exerted on the sample can be minimized by optimizing the imaging parameters, including the free amplitude, set point and scan rate. This technique is less invasive for nanobubble imaging compared to the contact mode, in which a soft isolated object can be destroyed or displaced by the tip during the scan of the sample.

\subsection{Spontaneous generation of nanobubbles on hydrophobic surfaces}

Tyrrell and Attard (2001) performed TMAFM imaging of hydrophobic surfaces in water. In their experiment they used glass surfaces exposed to dichlorodimethylsilane vapor for 3 min, giving a contact angle of $101^{\circ}$. The images show that the surfaces are covered with soft domains, apparently nanobubbles that do not occur in isolation with small surface coverage. They are close packed and irregular in cross section and literally cover the surface. Figure 1 presents a $1 \mu \mathrm{m}$ square image of the hydrophobic surface in water. Tyrrell and Attard (2001) also measured the phase image (not shown),

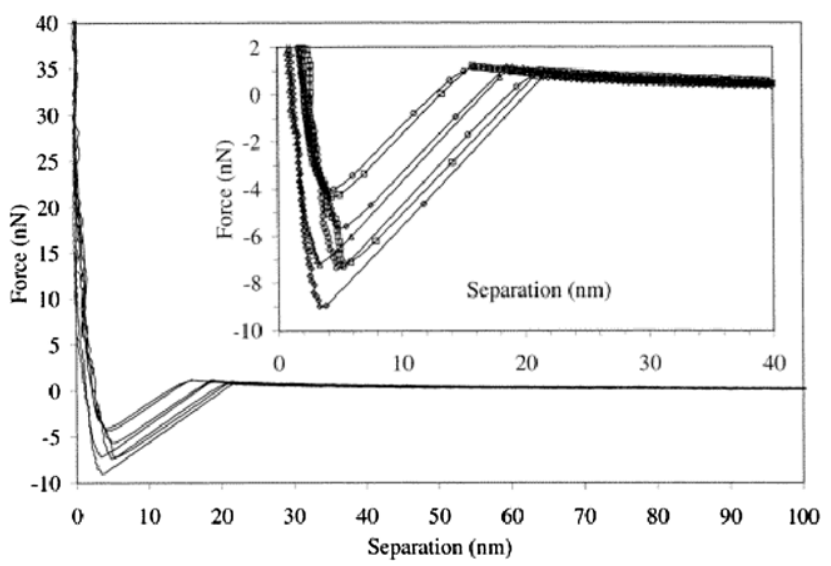

Figure 2. Force-separation curves for hydrophobic surfaces approaching a colloidal probe (silica sphere, radius $7.5 \mu \mathrm{m}$ ) at $\mathrm{pH}$ 5.6. The curves shown are for velocities $6.9,6.9,6.9,1.0,0.2$ and $0.2 \mu \mathrm{m}$ (from left to right). The inset is a zoom on the region where the jump occurs. Reprinted with permission from Tyrrell and Attard (2002). Copyright 2002 American Chemical Society.

which indicates that the corresponding features are composed of much softer material than the substrate and that it is not simply surface roughness that is being measured. Contact mode imaging of the same hydrophobic image in water was featureless and showed no nanobubbles. In contact mode the tip does not oscillate during the scan and it presses the bubbles so hard that it sweeps them aside. They also performed force measurements between a silica colloidal probe and the hydrophobic surface in water. Figure 2 shows plots of force versus separation between the surfaces for different velocities of approach. A jump into contact arises when the gradient of the attractive force exceeds the cantilever stiffness. When the approach velocity increases, the distance at which the particle and surface jump into contact decreases (Tyrrell and Attard 2002). They found that the jump distance is close to the height of the nanobubbles seen in the AFM images of the same sample. This measurement is further support that the bubbles are the origin of the hydrophobic interaction (Israelachvili and Pashley 1982, Tsao et al 1993, Parker et al 1994, Carambassis et al 1998, Attard 2003, Evans et al 2004).

Polystyrene (PS) coated glass or silicon surfaces are the simplest hydrophobic surfaces to prepare, in which one can also control the thickness of the coating. The surfaces are prepared by spin coating a PS solution with a given concentration in toluene. By controlling the concentration of the PS in toluene and the spin speed, the thickness can be adjusted to the desired value (Agrawal et al 2005).

Experiments carried out by Simonsen et al (2004) showed that nanobubbles behave as soft isolated objects on the PS coated surfaces. They also showed that coalescence can be induced during imaging of the bubbles with a high applied force. The coalescence of bubbles can be described by the Laplace-Young equation: $\Delta p=2 \gamma / R$, where $R$ is the radius of the bubbles, $\gamma$ is the surface tension and $\Delta p$ is the pressure difference between the inside and the outside of the sphere. For the air/water interface, the surface tension is constant. Therefore, the pressure difference is inversely proportional to 


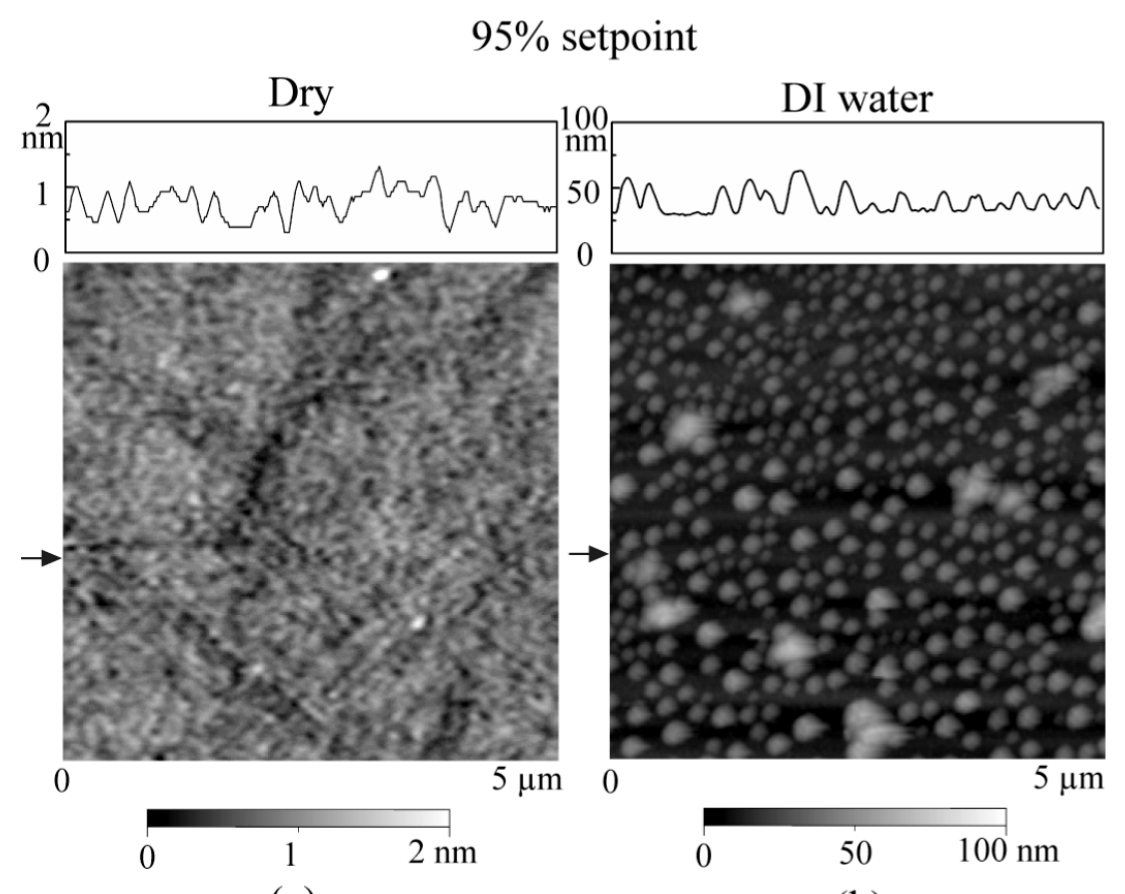

(a)

(b)

Figure 3. Comparison of images of a PS coated silicon wafer using tapping mode AFM in (a) air and (b) water (Bhushan et al 2008).

bubble radius. Small bubbles have greater internal pressure than larger bubbles so they are less stable than larger bubbles.

Bhushan et al (2008) studied the process of coalescence of nanobubbles during the imaging. They found that small bubbles can be easily moved and merged into larger ones. In their experiment they used a modified tip holder (Maali et al 2006) to directly provide piezo-excitation to the cantilever in the fluid. Figure 3 shows the image of a PS coated silicon surface in air and water. In air the image (figure 3(a)) is featureless, with an RMS roughness and peak to valley height of $0.21 \mathrm{~nm}$ and $2.3 \mathrm{~nm}$ respectively. The PS surface in water is covered by a spherical cap with a diameter of $200 \mathrm{~nm}$ and height of $20 \mathrm{~nm}$ (figure 3(b)). The RMS roughness and peak to valley height are $8.2 \mathrm{~nm}$ and $68.7 \mathrm{~nm}$ respectively-larger than that obtained in air.

Their measurements (Bhushan et al 2008) show that the nanobubbles are stable, except when the scan parameters are changed. They found that nanobubbles can coalesce with increasing scan load, as was previously reported (Steitz et al 2003, Simonsen et al 2004). They also found that the scan speed can affect nanobubble imaging.

Figure 4(a) (left) was obtained at a 95\% set point for a $5 \mu \mathrm{m} \times 5 \mu \mathrm{m}$ area scan. The scanning was performed twice on the central $2 \mu \mathrm{m} \times 2 \mu \mathrm{m}$ area with the same 95\% amplitude set point (figure 4(a) (right)). After that the $5 \mu \mathrm{m} \times 5 \mu \mathrm{m}$ area was imaged again with the $95 \%$ set point to get the image shown in figure 4(b) (right). Here we can see a lower density of nanobubbles in the central area, however with larger nanobubbles. The diameter and height of these larger nanobubbles increase to $420 \mathrm{~nm}$ and $55 \mathrm{~nm}$ respectively from the $200 \mathrm{~nm}$ and $20 \mathrm{~nm}$ measured in figure 4(a) (left). Therefore some nanobubbles must coalesce to generate the larger nanobubbles. The only difference between the $2 \mu \mathrm{m} \times$ $2 \mu \mathrm{m}$ central parts of figures 4(a) (right) and (left) is the scan speed. When working at the same scan rate, the scan speed in the $5 \mu \mathrm{m} \times 5 \mu \mathrm{m}$ area scan is one-and-a-half times greater than in the $2 \mu \mathrm{m} \times 2 \mu \mathrm{m}$ area scan. Assuming the mechanical power transmitted from the tip to the sample surface is constant during a certain period of time, the low scan speed implies higher power transfer at the same scan area than at high scan speed, and the nanobubbles suffer a greater disturbance. Therefore coalescence occurred even with the same amplitude set point. Bhushan et al (2008) have also investigated how the applied force affects nanobubble imaging. In their paper they presented details of the process of nanobubble coalescence while imaging under a high load. Here, we briefly reproduce their procedure for inducing nanobubble coalescence.

After applying a $90 \%$ set point scan over the whole $5 \mu \mathrm{m} \times 5 \mu \mathrm{m}$ area of figure 4(a) (right), a 95\% set point scan was performed over the full area and nanobubble images with a lower distribution density were obtained, as shown in figure 4(b) (left). With the lower bubble distribution density in figure 4(b) (left), it is possible to track certain bubbles' coalescence. In the central area containing the six numbered bubbles of figure 4(b) (left), a central $2 \mu \mathrm{m} \times 2 \mu \mathrm{m}$ area scan was performed twice with a $90 \%$ set point. Following this, a 95\% set point scan was performed over the full area, and a further nanobubble coalescence image was obtained, as shown in figure 4(b) (right). The diameter and height of the nanobubbles increase respectively from $550 \mathrm{~nm}$ and $77 \mathrm{~nm}$ in figure 4(b) (left) up to $690 \mathrm{~nm}$ and $100 \mathrm{~nm}$ in figure 4(b) (right). By comparing figure 4(b) (left) with figure 4(b) (right), one can find that, except for the six numbered bubbles 


\section{Scan speed influence}

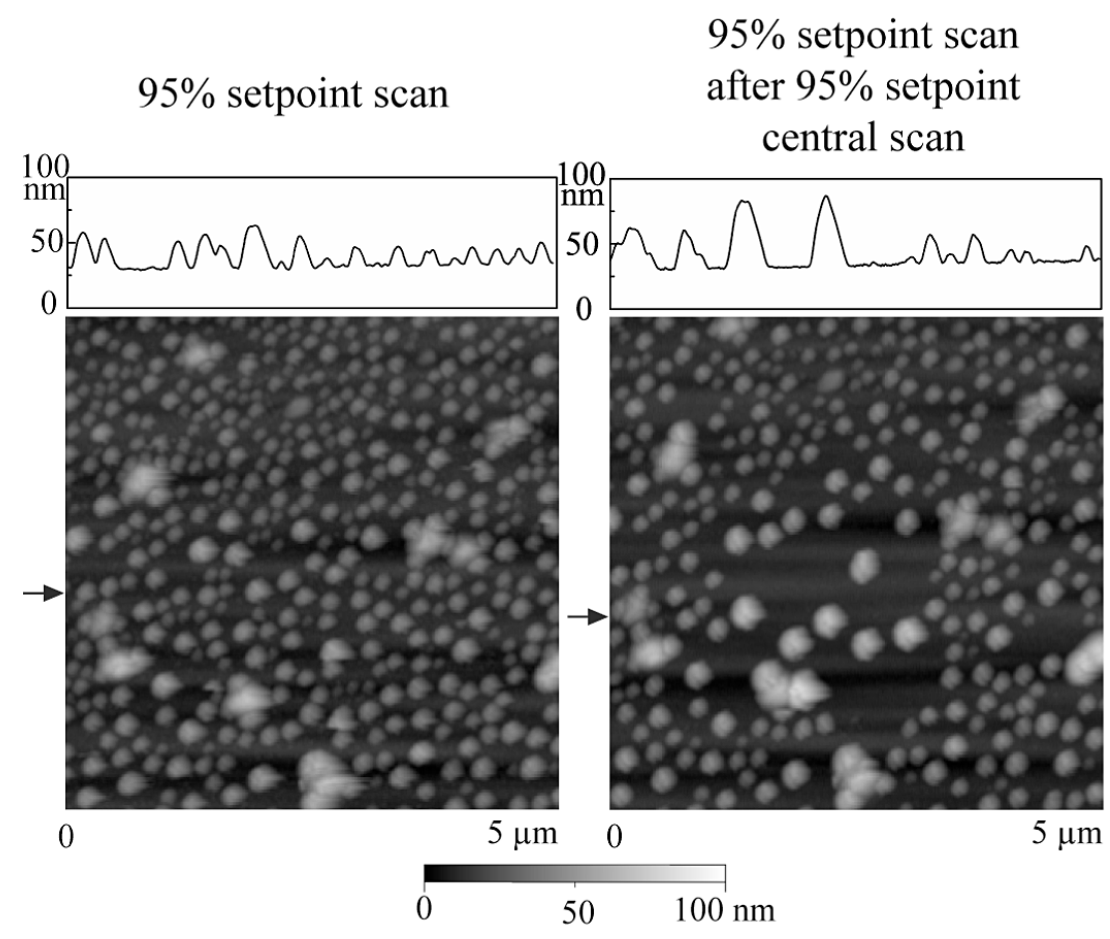

(a)

Further coalescence

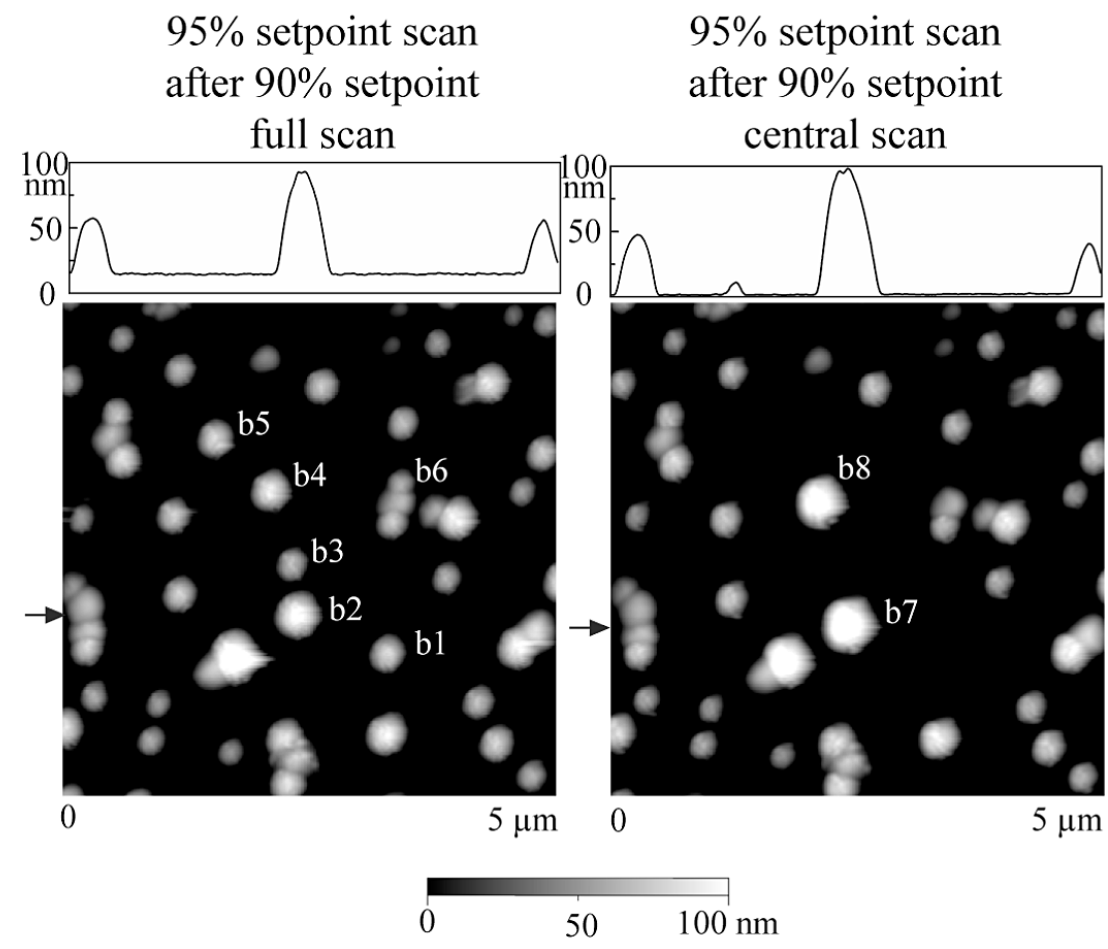

(b)

Figure 4. Sequence of images of nanobubbles on PS coated silicon wafer, obtained in the same $5 \mu \mathrm{m} \times 5 \mu \mathrm{m}$ scan area with (a) a 95\% amplitude set point scan (left); a 95\% amplitude set point scan preceded by scanning twice with a 95\% amplitude set point in the central $2 \mu \mathrm{m} \times 2 \mu \mathrm{m}$ area (right). Nanobubble coalescence is observed with a lower scan speed in the central area. (b) 95\% amplitude set point scan preceded by scanning with a 90\% amplitude set point over the full $5 \mu \mathrm{m} \times 5 \mu \mathrm{m}$ scan area (left); and 95\% amplitude set point scan preceded by scanning twice with a $90 \%$ amplitude set point in the central $2 \mu \mathrm{m} \times 2 \mu \mathrm{m}$ area (right). Further coalescence of nanobubbles is observed. Section profiles are taken at the locations shown by arrows in the AFM images (Bhushan et al 2008). 
in figure 4(b) (left), the sizes and locations of the other bubbles remained unchanged in figure 4(b) (right). Based on their locations, nanobubbles b1, b2 and b3 were believed to coalesce together and generate the larger bubble b7. Similarly, b4, b5 and b6 join together to generate bubble b8. More importantly, one can find that during nanobubble coalescence, small bubbles (b1, b3 and b5, b6) tend to move first and coalesce with (b2 and b4) generating larger ones. This should result because large bubbles have strong interactions with the surface due to their long contact line with the surface. Bhushan et al (2008) have verified this process of coalescence by performing a calculation of the quantity of gas molecules trapped in nanobubbles before and after coalescence.

From the above experiment, the nanobubbles are very sensitive to the scan parameters during imaging. To obtain original nanobubble images without movement and coalescence, a higher set point (corresponding to lower scan load) and higher scan rate are desirable. If the scan parameters are not chosen properly then the nanobubbles can be disturbed and they may coalesce.

Borkent et al (2010) have studied the contact angle of the nanobubbles as a function of their size. They used highly ordered pyrolytic graphite (HOPG, Mikromasch grade ZYA) as substrates to ensure clean, atomically flat surfaces. HOPG offers the advantage that by simply cleaning the sample (with adhesive tape) one gets a clean and atomically smooth surface. In their experiment they used a modified tip holder (Maali et al 2006), allowing them to work with a very small drive amplitude of the tip (of the order of a few nm). An example of their measurements is shown in figure 5. The topographic image as obtained by the cantilever tip is a combination of the nanobubble shape and the shape of the cantilever tip and therefore needs to be corrected for the tip shape. By determining the tip radius they could perform a deconvolution and obtain the radius of each nanobubble. From the image of the nanobubbles they calculated the contact angle for each nanobubble. Their experiment shows no noticeable dependence of the contact angle on the size of the nanobubbles. They also concluded that contamination may affect the measurements and lead to an increased value of the contact angle.

\subsection{Alcohol-water exchange}

Several groups have proposed a new process to generate nanobubbles by manipulating exchange between solutions of different gas solubilities, for example ethanol and water (Lou et al 2000, Zhang et al 2004, Hampton et al 2008, Zhang et al 2010, Guo et al 2012). This method is based on the principle that excess gas will accumulate near the solid surface due its different solubilities in water and ethanol. The surface was first exposed to ethanol, then water was injected to replace the ethanol and nanobubbles formed on the surface. Different alcohols lead to different size bubbles, the chain length of the alcohol used in the exchange affects the amount of gas present on the surface in the form of nanobubbles (Hampton et al 2008).

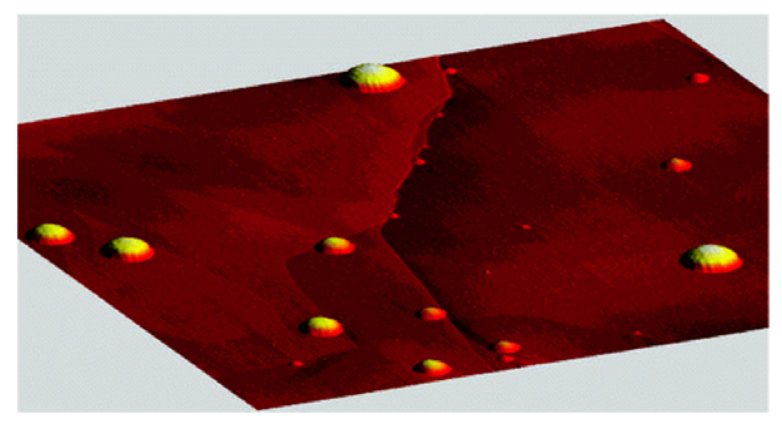

Figure 5. Image of surface nanobubbles present on a HOPG surface immersed in water. The size of the image is $2 \mu \mathrm{m} \times 2 \mu \mathrm{m}$. The nanobubbles show up as perfect spherical caps and have various sizes. They reside both at step edges and on atomically flat terraces. Apart from the step edges, the HOPG surface appears to be very smooth. Reprinted with permission from Borkent et al (2010). Copyright 2010 American Chemical Society.

With this process nanobubbles can form even on wetting surfaces such as mica (Zhang et al 2004). Experiments show that both the dissolved gas and temperature are important for the formation of nanobubbles. When the liquids were degassed the density of nanobubbles decreased compared with the density obtained by the non-degassed liquids (Zhang et al 2004). Zhang et al (2004) found also that the number of bubbles increases with increasing temperature. In another paper the same authors (Zhang et al 2005) showed that as the temperature of the sample increases from 28 to $42^{\circ} \mathrm{C}$, the lateral size of nanobubbles increases, reaching a maximum at about $37^{\circ} \mathrm{C}$, then decreases at a higher temperature. They explain their results using the temperature dependence of the gas solubility in water.

\subsection{Electrolytic generation of nanobubbles}

In electrochemical reactions, gas molecules are generated at electrode surfaces. Zhang et al (2006) and Yang et al (2009) have used this process to produce nanobubbles on the surface of highly oriented pyrolytic graphite (HOPG). When the HOPG surface is a negative electrode the gas that forms the nanobubbles is hydrogen, and if it is a positive electrode then the gas is oxygen. The setup used by Yang et al (2009) is shown in figure 6. In such an experiment the dissolved gases play a minor role in the surface nanobubbles. They found that an increase of voltage leads to an increase in the size of the nanobubbles. They found a correlation between the surface area and the volume growth rate of nanobubbles, suggesting that either the electrolytic gas emerges directly at the nanobubble surface or it emerges at the electrode surface and then diffuses through the nanobubbles. Replacement of pure water with water containing a small amount of sodium chloride $(0.01 \mathrm{M})$ gives qualitatively the same results.

\section{Boundary slip and drag force}

The no-slip boundary condition at a solid interface is at the center of our understanding of fluid mechanics (Lauga et al 2005, Neto et al 2005, Bocquet and Barrat 2007, Maali and 


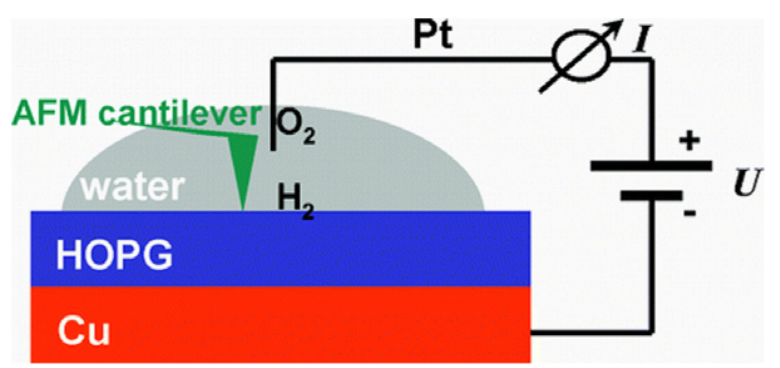

Figure 6. The experimental setup shows the HOPG sample placed on a copper plate. A platinum wire $0.25 \mathrm{~mm}$ is set ( $2 \mathrm{~mm}$ away) next to the AFM cantilever. The copper plate and the platinum wire were connected to a power supply. Reprinted with permission from Yang et al (2009). Copyright 2009 American Chemical Society.

Bhushan 2008, 2012, Bocquet and Charlaix 2010). The fluid velocity is assumed to be equal to the respective velocity of the surface. Recent experimental developments, allowing one to manipulate and control systems at micrometric and nanometric scales, have opened the way to testing if the no-slip boundary condition exists. Several experiments demonstrate an apparent slip of Newtonian liquids near hydrophobic solid surfaces.

The most popular method used to investigate boundary slip is the liquid drainage method using surface force apparatus (SFA) or AFM. The principle of this method is to measure the hydrodynamic drainage force between a sphere and a planar surface as a function of the separation distance when the surfaces approach each other (figure 7). Under the assumption of the no-slip boundary condition, the hydrodynamic drag force acting on the sphere is given as $F=\frac{6 \pi \eta R^{2}}{D} \frac{\mathrm{d} D}{\mathrm{~d} t}$, where $\eta$ is the viscosity, $R$ the radius of the sphere, $\frac{\mathrm{d} D}{\mathrm{~d} t}$ is the velocity of perpendicular approach of the sphere to the plane surface, and $D$ is the gap between the two surfaces. This expression is known as the Taylor equation.

In the presence of partial slip boundary conditions on the surfaces, Vinogradova (1995) calculated the drag force acting on the sphere when it approaches the wall. The calculation was made in the lubrication approximation, as for Taylor's solution. The solution has the form of Taylor's expression corrected by a factor $f^{*}$,

$$
F_{\mathrm{h}}=\frac{6 \pi \eta R^{2}}{D} \frac{\mathrm{d} D}{\mathrm{~d} t} f^{*}(D)
$$

The correction factor $f^{*}$ characterizes the slip boundary conditions. For the symmetric case where the slip occurs on both surfaces (sphere and flat wall), assuming the same amount of slip length $b$, the correction function is:

$$
f^{*}(D)=\frac{D}{3 b}\left[\left(1+\frac{D}{6 b}\right) \ln \left(1+\frac{6 b}{D}\right)-1\right] .
$$

This correction is usually used to describe the drainage of liquid between two hydrophobic surfaces.

For the asymmetric case, the slip occurs only on one surface, which is assumed to be hydrophobic, with the other surface being hydrophilic. Then the correction is

$$
f^{*}=\frac{1}{4}\left\{1+\frac{6 D}{4 b}\left[\left(1+\frac{D}{4 b}\right) \ln \left(1+\frac{4 b}{D}\right)-1\right]\right\} .
$$

Note here that the Vinogradova correction $f^{*}$ assumes a constant slip length $b$ that does not depend on the distance and shear rate. Experimentally, the hydrodynamic forces can be measured by multiplying the AFM cantilever's deflection
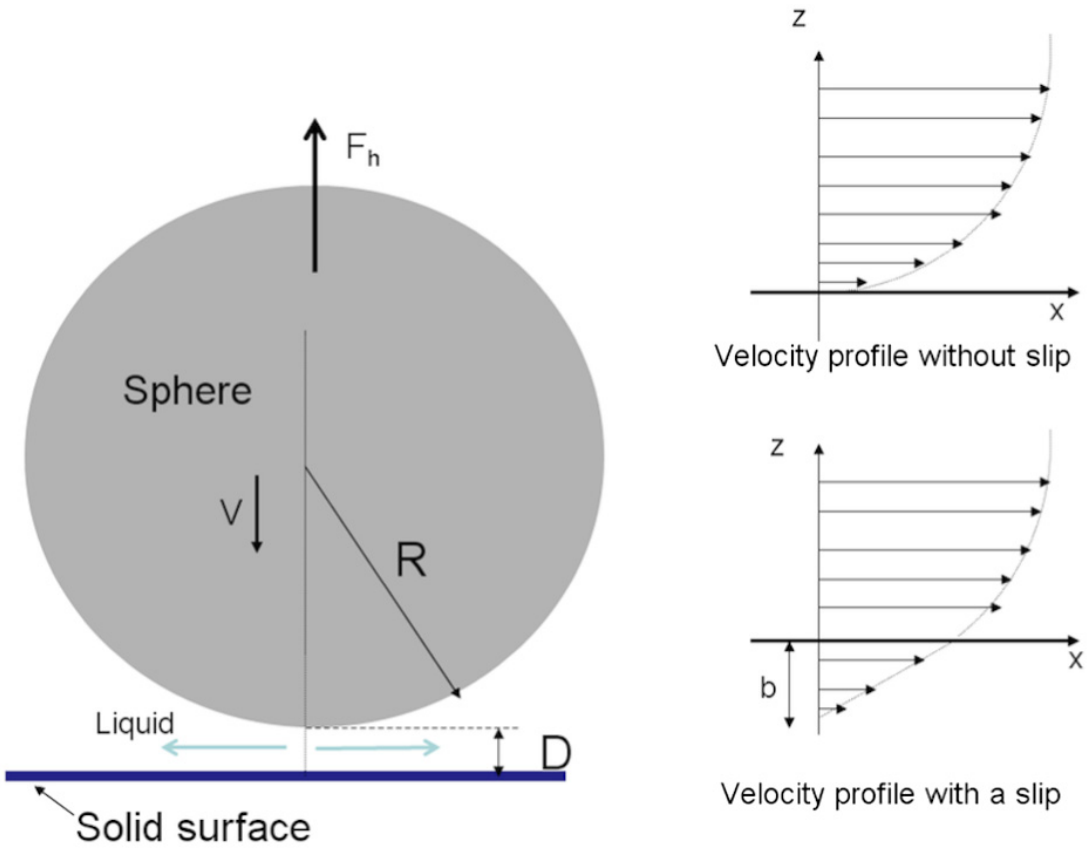

Velocity profile with a slip

Figure 7. Schematic of a sphere approaching a surface with a velocity $V$ and the velocity profile of fluid flow with and without boundary slip at the surfaces. The slip length $b$ characterizes the degree of boundary slip at the solid-liquid interface. To measure slip length, the hydrodynamic drag force $F_{\mathrm{h}}$ was recorded during the approach process. 
signal by the cantilever stiffness. The separation distance between the sphere and the solid surface can be obtained by adding the sphere displacement and the cantilever deflection. From the theoretical expression of the hydrodynamic force described above, the slip length can be obtained from the obtained hydrodynamic force and separation distance. Actually, it is believed that the no-slip boundary condition is satisfied for liquid flow on a hydrophilic surface (Honig and Ducker 2007). On a hydrophobic surface the slip length value is about tenth of a nanometer (Cottin-Bizonne et al 2005, Bocquet and Charlaix 2010). However, a larger value of slip length has been reported and the role of nanobubbles has been invoked, as is discussed below.

As we have shown in the previous section, nanobubbles appear spontaneously on hydrophobic surfaces (no-wetting surface). So, we may expect a large slip on such a surface. Tretheway and Meinhart (2004) calculated the slip length for fluid flow between two parallel plates. They reported that the slip length increases with increasing air gap thickness, assuming that air covers the wall continuously. The calculated slip length $b$ scales as $b=\frac{\eta_{w}}{\eta_{a}} h_{a}$, where $\eta_{w}$ and $\eta_{a}$ are the viscosity of water and air respectively, and $h_{a}$ is the thickness of the air gap.

For an intermittent surface coverage of nanobubbles, the slip length increases with increasing nanobubble height and surface fraction covered by nanobubbles, and is given by $b=$ $\phi \frac{\eta_{w}}{\eta_{a}} h_{b}$, where $\phi$ is the fraction of the sample surface covered by the nanobubbles and $h_{b}$ is the bubble height. At room temperature the viscosity for water and air are about $\eta_{w}=$ $1 \mathrm{~m} \mathrm{~Pa} \mathrm{~s}$ and $\eta_{a}=1 \mu \mathrm{Pa} \mathrm{s}$, respectively. Thus for a bubble on a hydrophobic surface having a coverage of $\phi=20 \%$ and height of $h_{b}=30 \mathrm{~nm}$ the expected slip length is about $300 \mathrm{~nm}$.

Using the surface force apparatus, Zhu and Granick (2001) reported a very large slip, up to $1 \mu \mathrm{m}$, on hydrophobic surface. The measured hydrodynamic drag force is up to 2-4 orders of magnitude less than expected by assuming the no-slip boundary condition. They also reported the slippage appearing only under confinement and above a critical shear rate. Steinberger et al (2008) have shown recently that elastohydrodynamic effects due to a non-perfectly rigid surface (such as glued mica thin films in SFA) exhibit this typical behavior. The existence of the slip length of water on smooth hydrophobic surfaces has been demonstrated by several groups using various methods, however the role played by the nanobubbles in determining the slip has not yet been completely resolved. Some new experiments cast doubt on whether the presence of nanobubbles could be responsible for the large slip length on a smooth hydrophobic surface.

Steinberger et al (2007) used surface force apparatus (SFA) to study liquid flow on superhydrophobic surfaces. Their experiments indicated the influence of liquid-gas menisci on the boundary conditions of the liquid flow. The trapped bubbles in the holes of the nanostructure act as an anti-lubricant and promote high friction. In fact, the curvature of the meniscus changes the streamlines of flow, which induces a transfer of momentum at the interface, thus increasing the friction instead of reducing it.

Steinberger et al (2007), Steinberger et al (2008) and Hyväluoma and Harting (2008) have studied the influence of

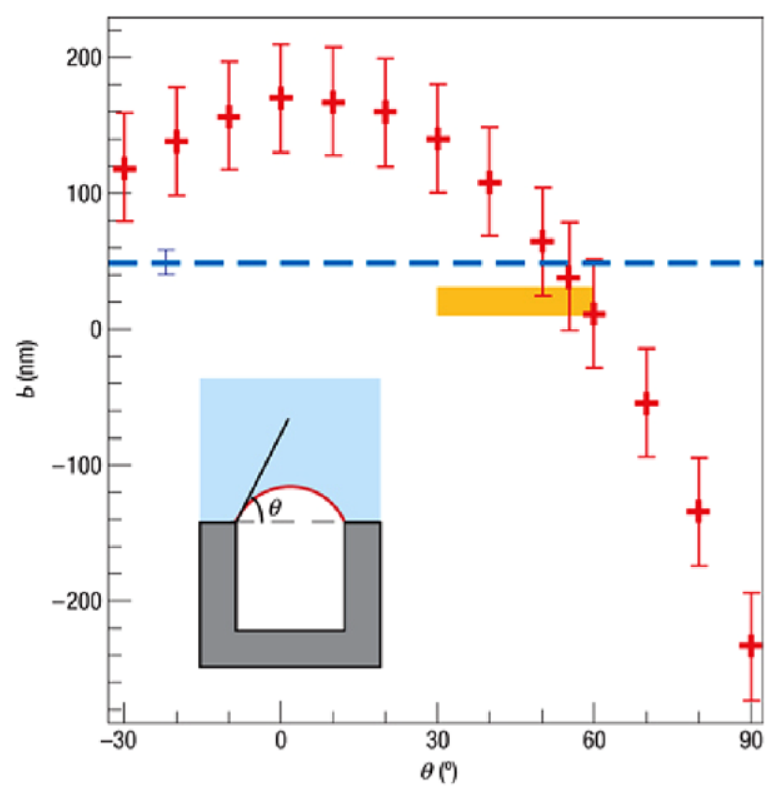

Figure 8. Evolution of the slip length $b$ with menisci shape. The dashed line corresponds to the numerical value in the hydrophilic case when the liquid fills the holes. Reprinted by permission from Macmillan Publisher Ltd Steinberger et al (2007). Copyright 2007.

the shape of the meniscus (contact angle of the bubbles) on the slip length. Their numerical calculations show a decrease of the effective slip length due to the meniscus curvature (figure 8). A large decrease of effective slip length is obtained for menisci that correspond to contact angles $\theta>45^{\circ}$. The obtained results are in agreement with the predictions of Richardson (1973) and Jansons (1988), which show that the boundary conditions for a shear-free interface become no-slip if the surface is sufficiently rough. The SFA experiment (Steinberger et al 2007) was corroborated by Finger and Johannsmann (2011), who performed an experiment where they used a quartz crystal microbalance (QCM) to study the slip length of water in the presence of bubbles. Their results suggest a reduction of the interfacial slippage in the presence of hemispherical nanobubbles.

Although there are some indications, as shown above, that bubbles can reduce the slip instead of increasing it, quantitative measurements are still lacking. It is really challenging to perform AFM measurement of the slip length at different positions of the hydrophobic surface and correlate the statistical value of the slip with the distribution of the nanobubbles on the surface.

\section{Drag force on micro-sized air pockets entrapped between pillars on microstructured surface}

As we have indicated in the previous section, the shape of the meniscus that forms nanobubbles has an important influence on liquid flow close to the interface. The atomic force microscope offers the opportunity to investigate this effect, since due to the small size of the probed area it allows measurement of the drag force at different positions on the sample where the flow lines behave differently. 


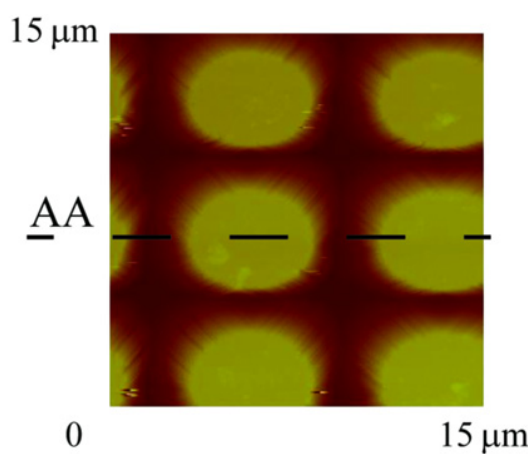

(a)

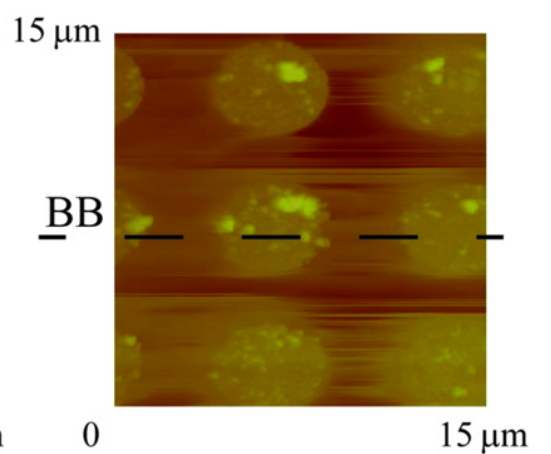

(b)

\section{Horizontal section - AA}

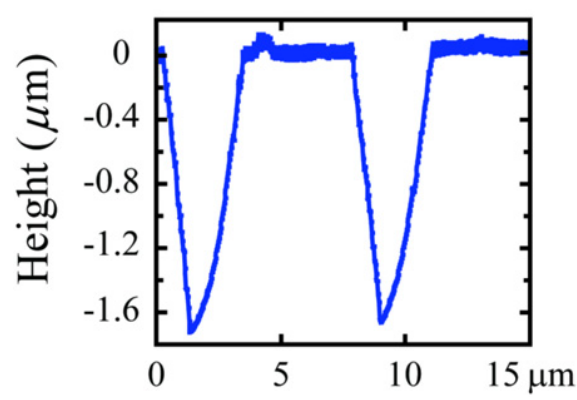

Figure 9. AFM images of the nanostructured surfaces in water. (a) Wenzel part (electric field applied), and (b) Cassie part. Note here that the height measured in (a) does not match the pillar height of $15 \mu \mathrm{m}$, instead it is $1.6 \mu \mathrm{m}$. This is because the height of the tip on the cantilever is $2 \mu \mathrm{m}$, which limits the depth the tip can penetrate, the measurements reflect the tip shape (Maali et al 2012).

Maali et al (2012) used colloidal probe atomic force microscopy (AFM) to measure the hydrodynamic drag force and slip length on a microstructured surface on which gas pockets can be trapped. The surfaces used in this experiment are made from silicon and consist of pillars $5.1 \mu \mathrm{m}$ in diameter ( $2 a$ ) spaced by $7.4 \mu \mathrm{m}$ (pitch, $L$ ). The height of the pillars is $15 \mu \mathrm{m}$. The surface was covered with a layer of tetrahydroperfluorodecyltrichlorosilane $\left(\mathrm{PF}_{3}\right)$, which makes it superhydrophobic. Optical measurements show that a water droplet forms a contact angle of $137^{\circ}$ on the surface. On one part of the surface the contact angle was reduced by applying a voltage of $20 \mathrm{~V}$ between the liquid and the substrate (irreversible electrowetting process). This process induces an irreversible transition from Cassie state wetting with air pocket formation to Wenzel state wetting, which means that water penetrates between the pillars, and the measured contact angle was $117^{\circ}$. Using this process a sample was created divided into two parts; in the first part the liquid at the interface forms a Cassie state, and in the second part the liquid at the interface forms a Wenzel state. Such a sample offers the advantage of switching during the experiment from the Cassie interface to the Wenzel interface just by translating the sample horizontally at two locations without changing samples.

The surfaces were imaged in liquid using an AFM in tapping mode with a tip of radius $10 \mathrm{~nm}$. The obtained images are shown in figure 9. The figure shows the images obtained in the Wenzel and Cassie parts and horizontal sections in both images. It was found that in the Wenzel part water penetrates between the pillars. However, the image of the Cassie part of the surface shows that water does not penetrate between the pillars, which leads to the trapping of gas pockets between the liquid and solid.

To measure hydrodynamic drag forces, drainage experiments were performed using the AFM in contact mode. The hydrodynamic drag force is given by the deflection of the cantilever as the sphere attached to the cantilever approaches the sample at a constant velocity. A spherical borosilicate particle with a diameter of $52.5 \mu \mathrm{m}$ was used. The benefits of using such a large particle are to minimize the hydrodynamic drag force due to the cantilever itself and to increase the hydrodynamic drag force due to the sphere. Also, with such a large sphere, a large separation distance of up to $D=2000 \mathrm{~nm}$ can be investigated while still remaining in the lubrication approximation $(D \ll R)$. The stiffness $k$ of the cantilever with a sphere attached was determined in situ using a flat silicon surface as a substrate (figure 10). First, the sphere is centered on a given pillar of the surface, then measurements of the hydrodynamic drag force as a function of the distance between the sphere and the surface of the pillar are performed. On each surface the measurement is made on several pillars to avoid problems of inhomogeneity and surface roughness of the pillars. The measured force on the structured surface is shown in figure 10(b). The measured force on the Wenzel part of the microstructured surface is smaller than the force on the flat silicon surface. This is easily understood by the fact that some of the flow occurs between pillars which are very high, and thus a lower hydrodynamic drag force is exerted on the sphere. For the Cassie part of this hydrophobic surface the 


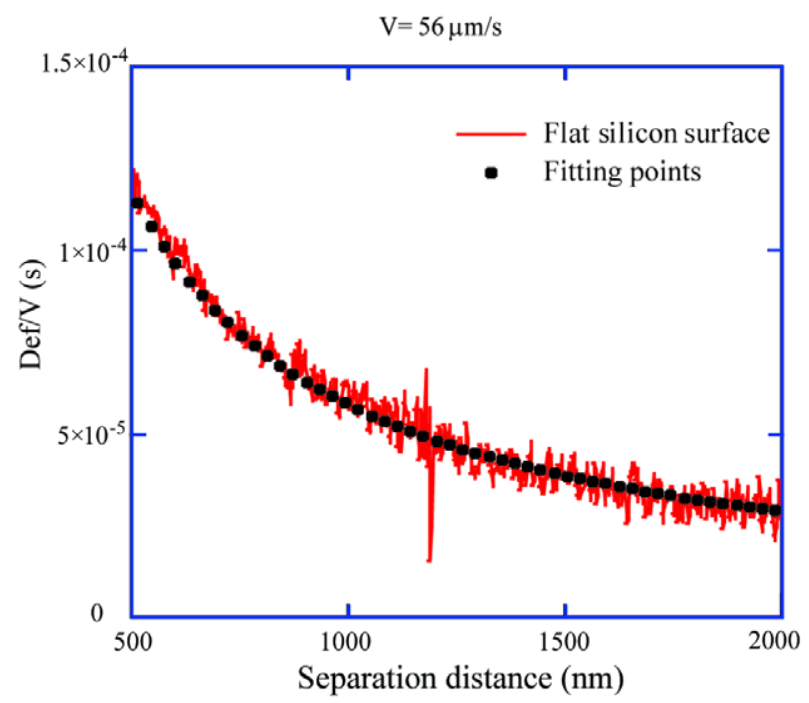

(a)

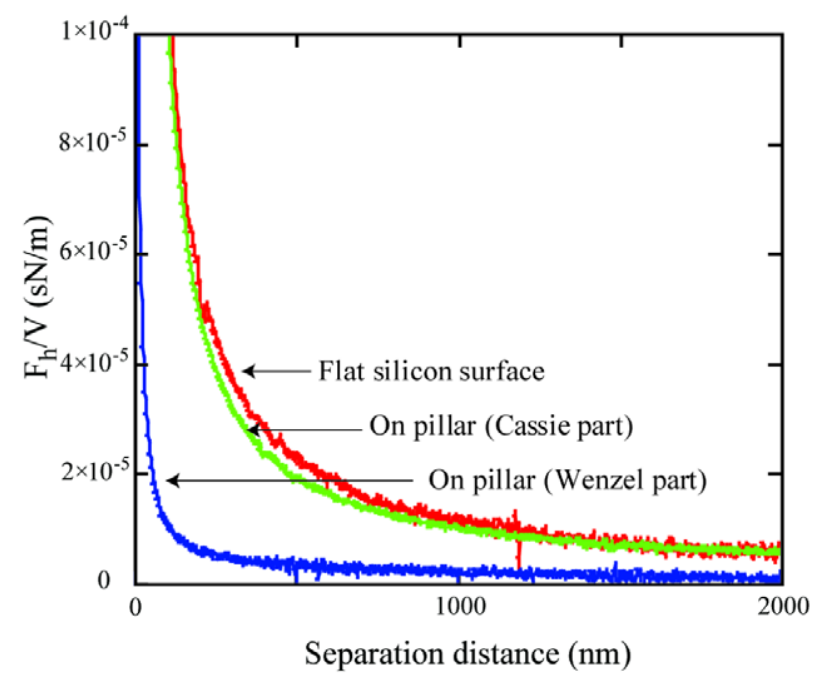

(b)

Figure 10. (a) The Def V for the flat silicon surface and the fitting points using equation (1). From the fit, $\frac{6 \pi \eta R^{2}}{k}=0.058 \mathrm{~nm} \mathrm{~s}$ is obtained, and thus a stiffness value of $k=0.19 \mathrm{~N} \mathrm{~m}^{-1}$, (b) the measured hydrodynamic drag force divided by velocity as a function of separation distance for the measurement on the flat silicon surface and on a pillar for both the Cassie part and the Wenzel part of the microstructured surface (Maali et al 2012).

force is roughly equal to the force measured on the flat silicon surface. This observation may seem strange. A large water slip on a hydrophobic surface is expected, thereby reducing the hydrodynamic drag force. In this case, the hydrodynamic drag force measured when the sphere is centered on a pillar of the Cassie part of the microstructured surface is not very small compared to the measured force on the silicon surface, which suggests a small slip.

To get a true picture of liquid flow on the Cassie surface, the flow between the pillars must be probed. The colloidal probe of an AFM offers this opportunity, since it allows the centering of the sphere between four pillars and then measures the local hydrodynamic drag force. The measured data are shown in figure 11(a). The data on the Cassie pillar and on

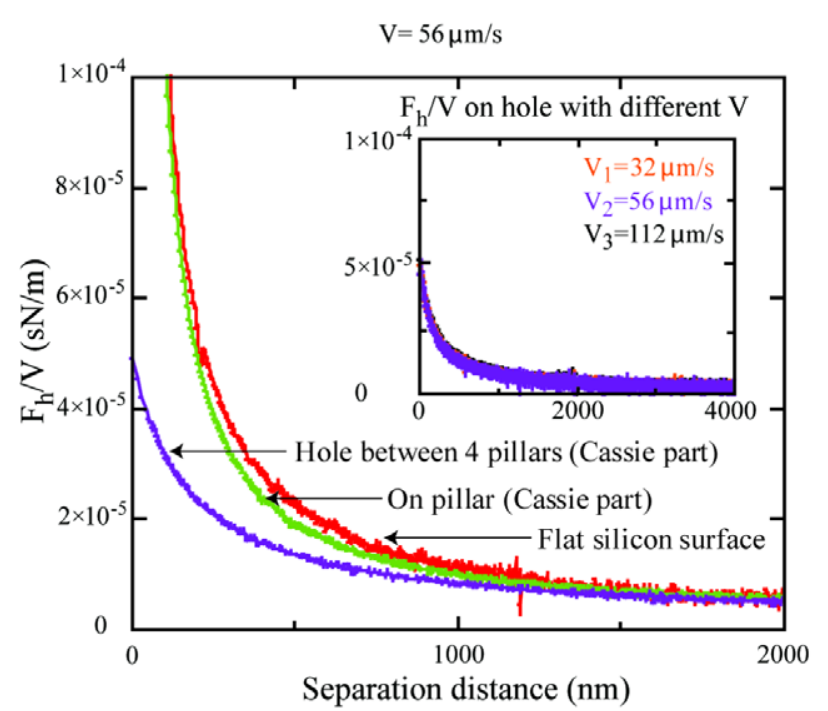

(a)

Flow Streamlines

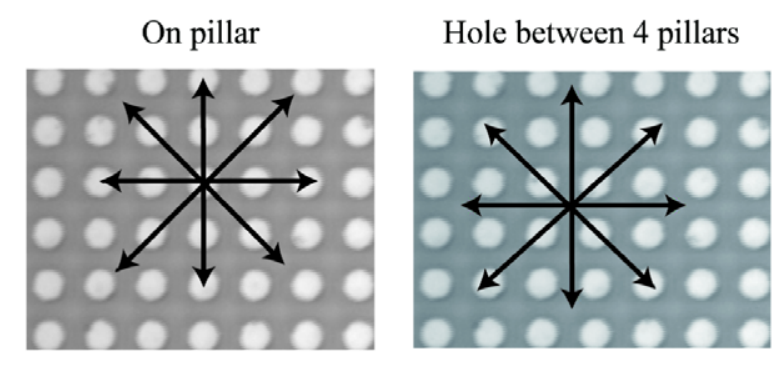

(b)

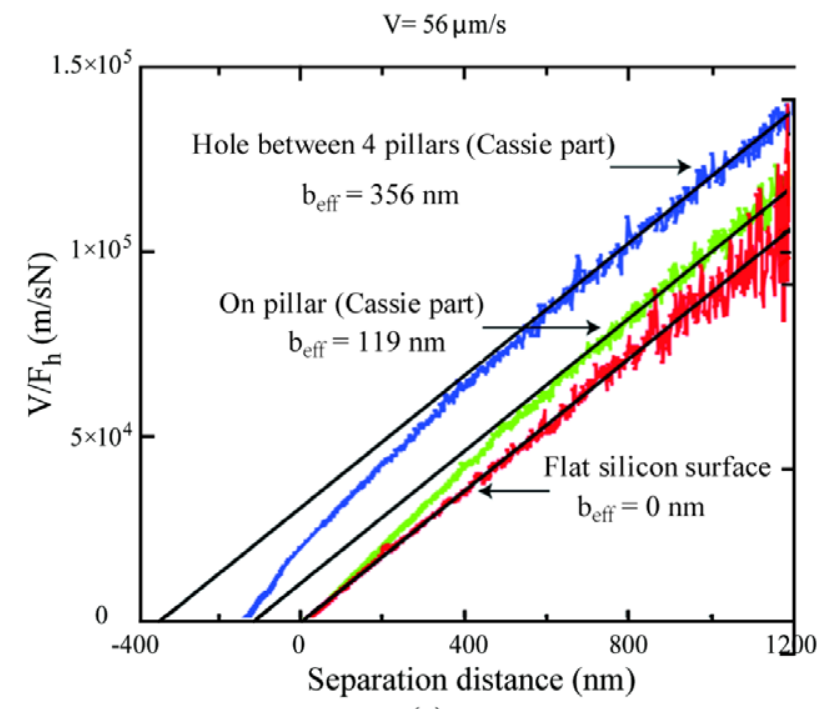

(c)

Figure 11. (a) The measured hydrodynamic drag force on flat silicon and on the Cassie part of the surface when the sphere is centered between four pillars and on a pillar of the microstructured surface. The data on the silicon surface are reported for comparison. In the inset, $F_{\mathrm{h}} / V$ is shown between four pillars for different velocities $V$ of approach: 32, 56, and $112 \mu \mathrm{m} \mathrm{s}^{-1}$. (b) Velocity divided by the hydrodynamic drag force $V / F_{\mathrm{h}}$ as a function of separation distance. The solid lines are linear extrapolations using the same slope for the three curves. The fitted values provide the slip length, $b_{\text {eff }}$, as indicated on each curve. (c) The flow streamlines close to the surface when the sphere is centered on a pillar and between four pillars (Maali et al 2012). 
the flat silicon surface are also reported in order to compare them. Between four pillars the edge of the sphere penetrates by an amount $\frac{(\sqrt{2} L-2 a)^{2}}{8 R} \approx 137 \mathrm{~nm}$. Thus, for measurements between four pillars the hard contact position is $137 \mathrm{~nm}$ below the pillar surface. In their analysis of the data they choose the pillar surface as the reference surface.

The inset shows the hydrodynamic drag force $F_{\mathrm{h}} / V$ for the Cassie part of the microstructured surface for different velocities $V$ of approach: 32, 56, and $112 \mu \mathrm{m} \mathrm{s}^{-1}$. The force is independent of the velocity, which means that the liquid-gas interface is not disturbed by the pressure induced by the approaching sphere. Furthermore, it was found that there is no capillary attraction of the interface to the sphere (data not shown). This is in agreement with the image presented in figure 9(b), which shows that the interface is underneath the reference plane formed by the pillar surface.

The value of the hydrodynamic drag force depends on the position where it is measured on the Cassie part of the microstructured surface. The value of the hydrodynamic drag force is lower when measured between pillars than when it is measured on a pillar itself. This can be explained by considering the streamline of the flow (see figure 11(c)). When the sphere is centered on the pillar, the liquid squeezed out by the approaching sphere will flow on a modulated topographic interface. For measurements between four pillars, part of the liquid squeezed out will flow in a straight line between the pillars over the liquid-gas interface. This part of the flow occurs with less friction, thus the total hydrodynamic drag force exerted on the sphere is reduced.

In order to accurately see the behavior of the water drainage and to extract the slip length, $b$, it is more convenient to present the data in the form $V / F_{\mathrm{h}}$, as was shown by Cottin-Bizonne et al (2005). In the limit of large separation distance $(D>b)$ :

$$
V / F_{\mathrm{h}}=\frac{D+b_{\mathrm{eff}}}{6 \pi \eta R^{2}}
$$

where $b_{\text {eff }}$ is the effective slip length. The advantage of presenting the data in the form of $V / F_{\mathrm{h}}$ is that the slip length appears as the position where the linear extrapolation of the curve $V / F_{\mathrm{h}}$ intercepts the distance axis. $V / F_{\mathrm{h}}$ as a function of separation distance is plotted in figure 11(b).

The plots obtained on the Cassie part of the microstructured surface (on pillars and between pillars) have the same slope as on the silicon surface. Furthermore, the measurements show that the flow when the sphere is centered between four pillars occurs with a larger slip than the flow when the sphere is centered on a pillar. The extracted slip length from fitting of the data gives $b_{\text {eff }}^{1}=119 \pm 30 \mathrm{~nm}$ and $b_{\text {eff }}^{2}=356 \pm 30 \mathrm{~nm}$ respectively on pillars and between four pillars, probed on the same area.

How can one relate these measurements to what is measured with other techniques? With the colloidal probe AFM one has access to semi-local values of the slip length on a heterogeneous surface, in contrast to other techniques which probe an average flow. Based on the fact that the 'pillar-centered' and the 'between-four-pillars' positions correspond to the extremes of the drag force at a given distance, one expects that the effective slip length at large distance is given by: $b_{\mathrm{eff}}^{\mathrm{av}}=\phi_{s} b_{\mathrm{eff}}^{1}+\left(1-\phi_{s}\right) b_{\mathrm{eff}}^{2}$, where $\phi_{s}$ is the solid fraction given as $\pi a^{2} / L^{2}=0.37$. For this value of $\phi_{s}, b_{\text {eff }}^{\mathrm{av}}=268 \pm 30 \mathrm{~nm}$.

This average slip length can be compared to theoretical models for a periodically patterned surface. The effective slip length on a two-dimensional array of pillars separated by a flat, free surface has been calculated by Davis and Lauga (2010), generalizing the result of Ybert et al (2007): $b_{\text {eff }}=$ $L\left(\frac{3}{16} \sqrt{\frac{\pi}{\phi_{s}}}-\frac{3}{2 \pi} \ln (1+\sqrt{2})\right)$. The corresponding value for our microstructured surface is $b_{\text {eff }}=926 \mathrm{~nm}$. This value greatly overestimates our measurements. The model does not take into account the curvature of the menisci, which significantly increases the interfacial friction. A more appropriate model to take into account this 3D character of the liquid boundary is Philip's calculation of the slip length on a periodic surface made of grooves oriented parallel to the flow (Philip 1972a, 1972b). Indeed, due to the lattice symmetry, the 3D undulation of the liquid-free surface due to the menisci disappears completely along lines lying midway between two adjacent pillar rows, whereas it is maximum along lines lying exactly on pillar centers. Therefore if one takes the groove size equal to the separation between two adjacent pillars ( $L-$ $2 a=2.3 \mu \mathrm{m}$ ), then Philip's model underestimates the actual friction on the grooves, but overestimates the friction between the grooves. The area fraction of the liquid-gas interface for this geometry is given by $\varphi_{g}=\frac{L-2 a}{L}=0.31$. The value of the slip length given by Philip (1972a), Lee et al (2008) is $b_{\text {eff }}=\frac{-L}{\pi} \ln \left[\cos \left(\frac{\pi}{2} \varphi_{g}\right)\right]=291 \mathrm{~nm}$. This calculated value is close to the average slip length measured in their experiment (Maali et al 2012).

These measurements show the important role played by the shape of the interface on the liquid flow. Furthermore, in the Cassie part of the microstructured surface a larger hydrodynamic drag force was measured on the pillar than between pillars, although the area probed by the flow is of the order of the lattice unit cell. Corresponding values of semi-local, coarse-grained slip lengths were extracted, the average value of which gives a reliable estimate of the effective slip length. The effective slip length is described well by the Philip's equation for grooves parallel to the flow, which shows that an important consequence of the meniscus curvature is to favor flow between adjacent rows of pillars.

\section{Conclusion and outlook}

Nanobubbles are very soft gas domains that form on the solid-liquid interface. They can be formed spontaneously on hydrophobic surfaces. They can be also prepared by electrolysis and by alcohol-water exchange. Nanobubbles are very stable to dissolution and can be moved by the AFM tip, while smaller ones can coalesce into larger ones.

It is generally believed that the presence of nanobubbles can explain liquid slip at the interface and the resulting lower drag. However, some new experiments indicate the opposite. These experiments demonstrated that the shape of the menisci that form the bubbles on the interface play an important role 
in the liquid flow. The bubbles can act as an anti-lubricant and thus increase the drag instead of reducing it.

Although the field of bubbles and nanobubbles on surfaces has been extensively studied, as shown in this review, still many challenges remain. Whether surface contaminants are responsible for preventing gas diffusion outside the bubble and thus influencing bubble stability is not well understood. The process of nanobubble nucleation on the surface is another task that would be of interest to study.

From the nanobubble research, micropancakes have been discovered (Zhang et al 2007b, Seddon et al 2010). These consist of a very thin film of gas having a height of a few nanometers that are also very wide, up to a few micrometers. Their process of formation and decay is an open field of research. They may be a suitable system to investigate drag reduction using a colloidal probe technique, since the effect of the menisci will be smaller due the large lateral size of the micropancakes.

\section{References}

Agrawal A, Park J, Ryu D Y, Hammond P T, Russell T P and McKinley G H 2005 Controlling the location and spatial extent of nanobubbles using hydrophobically nanopatterned surfaces Nano Lett. 5 1751-6

Attard P 2003 Nanobubbles and the hydrophobic attraction $A d v$. Colloid Interface Sci. 104 75-91

Bhushan B, Wang Y and Maali A 2008 Coalescence and movement of nanobubbles studied with tapping mode AFM and tip-bubble interaction analysis J. Phys.: Condens. Matter 20485004

Bocquet L and Barrat J-L 2007 Flow boundary conditions from nano- to micro-scales Soft Matter 3 685-93

Bocquet L and Charlaix E 2010 Nanofluidics, from bulk to interfaces Chem. Soc. Rev. 39 1073-95

Borkent B M, de Beer S, Mugele F and Lohse D 2010 On the shape of surface nanobubbles Langmuir 26 260-8

Carambassis A, Jonker L C, Attard P and Rutland M W 1998 Forces measured between hydrophobic surfaces due to a submicroscopic bridging bubble Phys. Rev. Lett. 805357

Cottin-Bizonne C, Cross B, Steinberger A and Charlaix E 2005 Boundary slip on smooth hydrophobic surfaces: intrinsic effects and possible artifacts Phys. Rev. Lett. 94056102

Craig V S J 2011 Very small bubbles at surfaces-the nanobubble puzzle Soft Matter 7 40-8

Davis A M J and Lauga E 2010 Hydrodynamic friction of fakir-like superhydrophobic surfaces J. Fluid Mech. 661 402-11

de Gennes P G 2002 On fluid/wall slippage Langmuir 183413

Evans D R, Craig V S J and Senden T J 2004 The hydrophobic force: nanobubbles or polymeric contaminant? Physica A 339 101-5

Finger A and Johannsmann D 2011 Hemispherical nanobubbles reduce interfacial slippage in simple liquids Phys. Chem. Chem. Phys. 13 18015-22

Guo W, Shan H, Guan M, Gao L, Liu M and Dong Y 2012 Investigation on nanobubbles on graphite substrate produced by the water- $\mathrm{NaCl}$ solution replacement Surf. Sci. 606 1462-6

Hampton M A, Donose B C and Nguyen A V 2008 Effect of alcohol-water exchange and surface scanning on nanobubbles and the attraction between hydrophobic surfaces $J$. Colloid Interface Sci. 325 267-74

Honig C D F and Ducker W A 2007 No-slip hydrodynamic boundary condition for hydrophilic particles Phys. Rev. Lett. 98028305

Hyväluoma J and Harting J 2008 Slip flow over structured surfaces with entrapped microbubbles Phys. Rev. Lett. 100246001
Israelachvili J and Pashley R 1982 The hydrophobic interaction is long range, decaying exponentially with distance Nature $300341-2$

Jansons K M 1988 Determination of the macroscopic (partial) slip boundary condition for a viscous flow over a randomly rough surface with a perfect slip microscopic boundary condition Phys. Fluids 31 15-7

Karpitschka S, Dietrich E, Seddon J R T, Zandvliet H J W, Lohse D and Riegler H 2012 Nonintrusive optical visualization of surface nanobubbles Phys. Rev. Lett. 109066102

Lauga E and Brenner M P 2004 Dynamic mechanisms for apparent slip on hydrophobic surfaces Phys. Rev. E 70026311

Lauga E, Brenner M P and Stone H A 2005 Microfluidics: the no-slip boundary condition Springer Handbook of Experimental Fluid Dynamics (New York: Springer) pp 1219-40

Lee C, Choi C H and Kim C J 2008 Structured surfaces for a giant liquid slip Phys. Rev. Lett. 101064501

Lou S-T, Ouyang Z-Q, Zhang Y, Li X J, Hu J, Li M-Q and Yang F-J 2000 Nanobubbles on solid surface imaged by atomic force microscopy J. Vac. Sci. Technol. B 18 2573-5

Maali A and Bhushan B 2008 Nanorheology and boundary slip in confined liquids using atomic force microscopy J. Phys.: Condens. Matter 20315201

Maali A and Bhushan B 2012 Measurement of slip length on superhydrophobic surfaces Phil. Trans. R. Soc. A 370 2304-20

Maali A, Hurth C, Cohen-Bouhacina T, Couturier G and Aime J P 2006 Improved acoustic excitation of atomic force microscope cantilevers in liquids Appl. Phys. Lett. 88163504

Maali A, Pan Y, Bhushan B and Charlaix E 2012 Hydrodynamic drag-force measurement and slip length on microstructured surfaces Phys. Rev. E 85066310

Neto C, Evans D R, Bonaccurso E, Butt H-J and Craig V S J 2005 Boundary slip in Newtonian liquids: a review of experimental studies Rep. Prog. Phys. 682859

Parker J L, Claesson P M and Attard P 1994 Bubbles, cavities, and the long-ranged attraction between hydrophobic surfaces J. Phys. Chem. 34 8468-80

Philip J 1972a Flows satisfying mixed no-slip and no-shear conditions Z. Angew. Math. Phys. 23353

Philip J 1972b Integral properties of flows satisfying mixed no-slip and no-shear conditions Z. Angew. Math. Phys. 23960

Poynor A, Hong L, Robinson I K, Granick S, Zhang Z and Fenter P A 2006 How water meets a hydrophobic surface Phys. Rev. Lett. 97266101

Richardson S 1973 No-slip boundary condition J. Fluid Mech. 59 707-19

Seddon J R T, Bliznyuk O, Kooij E S, Poelsema B, Zandvliet H J W and Lohse D 2010 Dynamic dewetting through micropancake growth Langmuir 269640

Seddon J R T and Lohse D 2011 Nanobubbles and micropancakes: gaseous domains on immersed substrates J. Phys.: Condens. Matter 23133001

Simonsen A C, Hansen P L and Klosgen B 2004 Nanobubbles give evidence of incomplete wetting at a hydrophobic interface J. Colloid Interface Sci. 273 291-9

Steinberger A, Cottin-Bizonne C, Kleimann P and Charlaix E 2007 High friction on a bubble mattress Nature Mater. 6 665-8

Steinberger A, Cottin-Bizonne C, Kleimann P and Charlaix E 2008 Nanoscale flow on a bubble mattress: effect of surface elasticity Phys. Rev. Lett. 100134501

Steitz R, Gutberlet T, Hauss T, Klosgen B, Krastev R, Schemmel S, Simonsen A C and Findenegg G H 2003 Nanobubbles and their precursor layer at the interface of water against a hydrophobic substrate Langmuir 19 2409-18

Switkes M and Ruberti J W 2004 Rapid cryofixation/freeze fracture for the study of nanobubbles at solid-liquid interfaces Appl. Phys. Lett. 84 4759-61 
Tretheway D C and Meinhart C D 2004 A generating mechanism for apparent fluid slip in hydrophobic microchannels Phys. Fluids 16 1509-15

Tsao Y-H, Evans D F and Wennerström H 1993 Long-range attractive force between hydrophobic surfaces observed by atomic force microscopy Science 262 547-50

Tyrrell J W G and Attard P 2001 Images of nanobubbles on hydrophobic surfaces and their interactions Phys. Rev. Lett. 87176104

Tyrrell J W G and Attard P 2002 Atomic force microscope images of nanobubbles on a hydrophobic surface and corresponding force-separation data Langmuir 18 160-7

Vinogradova O I 1995 Drainage of a thin liquid film confined between hydrophobic surfaces Langmuir 11 2213-20

Wang Y and Bhushan B 2010 Boundary slip and nanobubble study in micro/nanofluidics with atomic force microscope Soft Matter 6 29-66

Yang S, Tsai P, Kooij E S, Prosperetti A, Zandvliet H J W and Lohse D 2009 Electrolytically generated nanobubbles on highly orientated pyrolytic graphite surfaces Langmuir 25 1466-74

Ybert C, Barentin C, Cottin-Bizonn C, Joseph P and Bocquet L 2007 Achieving large slip with superhydrophobic surfaces: scaling laws for generic geometries Phys. Fluids 19123601
Zhang X H, Khan A and Ducker W A 2007a A nanoscale gas state Phys. Rev. Lett. 98136101

Zhang X-H, Li G, Wu Z-H, Zhang X-D and Hu J 2005 Effect of temperature on the morphology of nanobubbles at mica/water interface Chin. Phys. 14 1774-8

Zhang X H, Quinn A and Ducker W A 2008 Nanobubbles at the interface between water and a hydrophobic solid Langmuir 24 4756-64

Zhang X H, Zhang X D, Lou S T, Zhang Y, Li M, Sun J, Li X and $\mathrm{Hu} \mathrm{J} 2004$ Degassing and temperature effects on the formation of nanobubbles at the mica/water interface Langmuir 203813

Zhang X H, Zhang X, Sun J, Zhang Z, Li G, Fang H, Xiao X, Zeng X and Hu J 2007b Detection of novel gaseous states at the highly oriented pyrolytic graphite-water interface Langmuir 23 1778-83

Zhang L, Zhang X, Zhang Y, Hu J and Fang H 2010 The length scales for stable gas nanobubbles at liquid/solid surfaces Soft Matter 6 4515-9

Zhang L, Zhang Y, Zhang X, Li Z, Shen G, Ye M, Fan C, Fang $\mathrm{H}$ and $\mathrm{Hu} \mathrm{J} 2006$ Electrochemically controlled formation and growth of hydrogen nanobubbles Langmuir 22 8109-13

Zhu Y and Granick S 2001 Rate-dependent slip of Newtonian liquid at smooth surfaces Phys. Rev. Lett. 87096105 